EPJ Web of Conferences 59, 13012 (2013)

DOI: $10.1051 /$ epjconf/20135913012

(C) Owned by the authors, published by EDP Sciences, 2013

\title{
Development of time-of-flight neutron detector with fast-decay and low-afterglow scintillator for fast ignition experiment
}

\author{
T. Nagai ${ }^{1}$, Y. Arikawa ${ }^{1}$, H. Hosoda ${ }^{1}$, Y. loka ${ }^{1}$, A. Hasegawa ${ }^{2}$, K. Wada ${ }^{2}$, \\ S. Takaoku ${ }^{2}$, M. Takata ${ }^{2}$, K. Noritake ${ }^{2}$, Y. Minami ${ }^{1}$, K. Watanabe ${ }^{1}$, \\ K. Yamanoi ${ }^{1}$, H. Nakamura ${ }^{1}$, T. Watari ${ }^{1}$, M. Cadatal-Raduban ${ }^{1}$, T. Shimizu ${ }^{1}$, \\ N. Sarukura ${ }^{1}$, M. Nakai ${ }^{1}$, T. Norimatsu ${ }^{1}$ and H. Azechi ${ }^{1}$ \\ ${ }^{1}$ Institute of Laser Engineering, Osaka University, Japan \\ ${ }^{2}$ Division of Sustainable Energy and Environmental Engineering, Graduate School of \\ Engineering, Osaka University, Japan
}

\begin{abstract}
A fast-decay and low-afterglow liquid scintillator was developed for the fast ignition experiment at the Institute of Laser Engineering (ILE). The liquid scintillator was coupled to a gated photomultiplier (PMT), and the gating performance under high-intensity $\gamma$-rays was experimentally checked. In 2010, a detector with a high detection efficiency of $10^{-4}$ was developed and installed in this experiment. The neutron yield in the fast heating experiment was successfully measured using this detector.
\end{abstract}

\section{INTRODUCTION}

Fast ignition is considered to be one of the most promising alternative ignition schemes for realizing inertial confinement fusion. In this scheme, the imploded core is heated by using an electron beam generated by an ultra-high intensity laser; simultaneously, high-intensity high-energy x-ray (called as $\gamma$ rays) are generated. Neutron detection in the fast ignition process is very difficult owing to the large background signals, especially the long tail of the scintillation signal (afterglow), originating from the prompt $\gamma$-rays. Therefore, it is necessary to develop a fast-response and low-afterglow scintillator. R. Lauck et al. and C. Stoeckl et al. have already reported very low afterglow liquid scintillators [1, 2]. This scintillator consisted of a xylene solution with a fluorescent dye quenched by oxygen gas. In the fast ignition experiment carried out at the Institute of Laser Engineering (ILE), Osaka University, a highsensitivity neutron detector, typically with detection efficiency $>10^{-4}$, was required in order to detect neutrons at a relatively lower yield of $\sim 10^{5}$. Thus, the detector should be placed close to the target, typically $\sim 3 \mathrm{~m}$ away, which is much shorter than that in the OMEGA laser [2]. In addition, it will be necessary to improve the above mentioned liquid scintillator to realize a faster and higher light yield.

The gating technique for strong $\gamma$-rays is a difficult issue. A microchannel plate based photomultiplier (MCP-PMT), a widely used gated PMT, and a gate-mesh attached on the photo cathode cannot shield strong $\gamma$-rays, because $\gamma$-rays directly interact in the MCP, and thus, a large number of electrons are generated [2]. In this study, the improved liquid scintillator was developed, and a neutron detector was developed by using a PMT with a gating module in the dynode bleeder circuit [3, 4]. In 2010, the detector was installed in the fast ignition experiment at the GEKKO XII and LFEX lasers at the ILE, and the neutron signal was successfully observed [5].

This is an Open Access article distributed under the terms of the Creative Commons Attribution License 2.0, which permits unrestricted use, distribution, and reproduction in any medium, provided the original work is properly cited. 


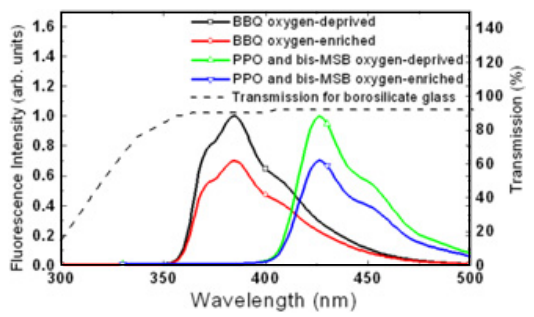

Figure 1. Comparison of fluorescence spectrum of BBQ liquid scintillators and PPO + bis-MSB liquid scintillators with and without $\mathrm{O}_{2}$.

(a)

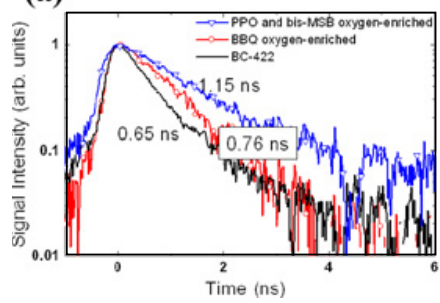

(b)

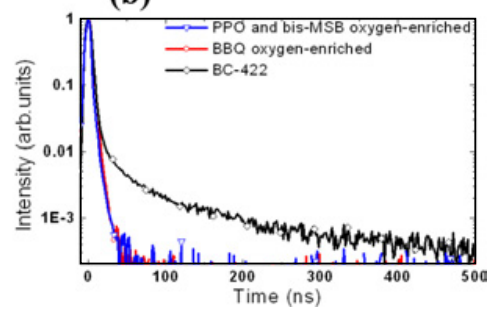

Figure 2. Comparison of afterglow of BBQ (with and without $\mathrm{O}_{2}$ ), $\mathrm{PPO}+$ bis-MSB (with and without $\mathrm{O}_{2}$ ), and BC-422 [8]. (a) Fast component decay curve excited by a UV short pulse and observed by a streak camera, fitted decay curve (dotted) and decay time, and (b) slow component decay curve excited by a $\gamma$-ray from the ${ }^{60}$ Co source and detected by the time-correlated single photon counting technique [9].

\section{FAST-RESPONSE LIQUID SCINTILLATOR}

We improved upon the previously used scintillator (mentioned above) by using BBQ (4, $4^{\prime \prime \prime}-$ bis [(2butyloctyl)oxy]-1, 1': $4^{\prime}, 1^{\prime \prime}: 4^{\prime \prime}, 1^{\prime \prime \prime}$-quarterphenyl) organic fluorescent dye to realize fast decay and a low afterglow. This dye has a shorter emission wavelength; this is preferable because the decay time is generally proportional to the cube of the wavelength. The emission wavelength, however, should be longer than the transmission edge of the borosilicate glass or acryl $(\sim 350 \mathrm{~nm})$ used in the light guide or photo window of the PMT and should meet PMT sensitivity $(300 \sim 500 \mathrm{~nm})$ [6]. Figure 1 shows a comparison of the emission wavelength of the developed liquid scintillator with that of other scintillators with different compositions.

Figure 2 shows a comparison of the afterglow of various decay curves [1]. The details are discussed in a previous study [7]. From the figure, the $\mathrm{O}_{2}$-enriched BBQ liquid scintillator is observed to have the best response and photon yield.

\section{DETECTOR DESIGN}

A PMT with a larger window diameter is preferable for realizing high photon collecting efficiency. However, the diameter of an MCP-PMT is generally small; therefore, a dynode PMT with a larger photo window was selected.

Furthermore, the gating function in the MCP-PMT with a gate mesh on the cathode cannot block strong $\gamma$-rays perfectly, because the $\gamma$-rays directly hit the MCP and generate photoelectrons in it, even though the scintillation light can be blocked. The gating function in the dynode bleeder circuit is robust because electron current in PMT can be stopped by reverse bias [3]. The gating performance of these two types of gated PMT was tested under high-intensity $\gamma$-rays, as shown in Fig. 3. The black lines 


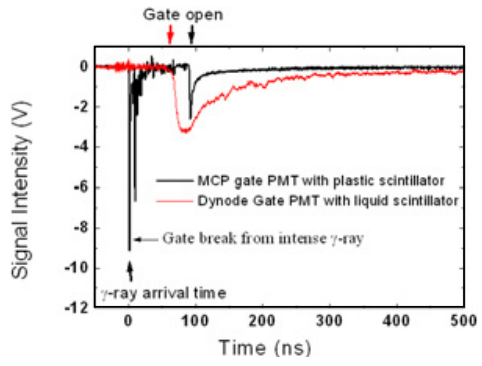

Figure 3. Gating performance of two types of gated PMT under high-intensity $\gamma$-rays.

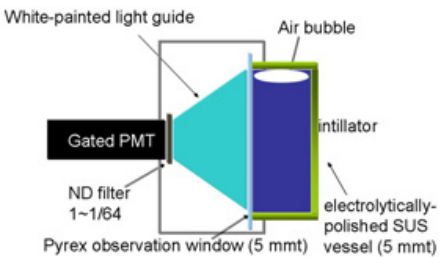

Figure 4. Schematic of our neutron TOF detector.

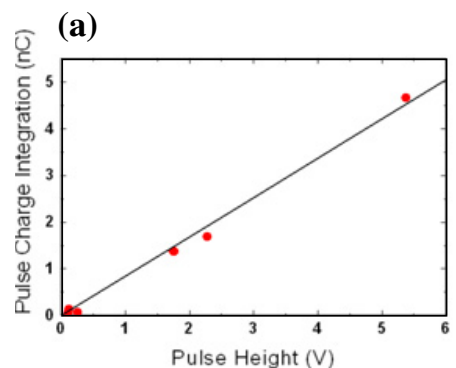

(b)

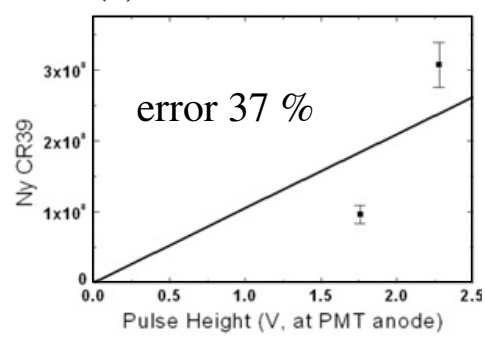

Figure 5. (a) Pulse linearity check, (b) relation between neutron yield and pulse height.

indicate the signal from the detector with a gated MCP-PMT with the BC-422Q plastic scintillator and the red line, that from the detector with a liquid scintillator with a dynode gating PMT. The latter showed good suppression of $\gamma$-rays. Furthermore, the light coupling efficiency from the scintillator to PMT was much higher, and it was confirmed that this enabled the detection of a neutron signal from ${ }^{252} \mathrm{Cf}$ by using non-gated PMT.

A liquid scintillator with a volume of $2.5 \mathrm{~L}(18 \mathrm{~cm} \varphi, 10 \mathrm{cmt})$ was connected to the gated PMT via a UV-transmitting acryl light guide that was painted white. The light yield at the PMT cathode was adjusted by neutral density filters. Figure 4 shows the schematic of our neutron time-of-flight (TOF) detector.

\section{NEUTRON MEASUREMENT IN FAST IGNITION EXPERIMENT}

The detector was set $3.1 \mathrm{~m}$ away from the center of the target chamber at an angle of $102^{\circ}$ from the LFEX laser injection. Before diagnosing the fast ignition shots, the pulse shape linearity was checked experimentally, as shown in Fig. 5(a). In the fast ignition shots, a $\gamma$-n background was found to exist 


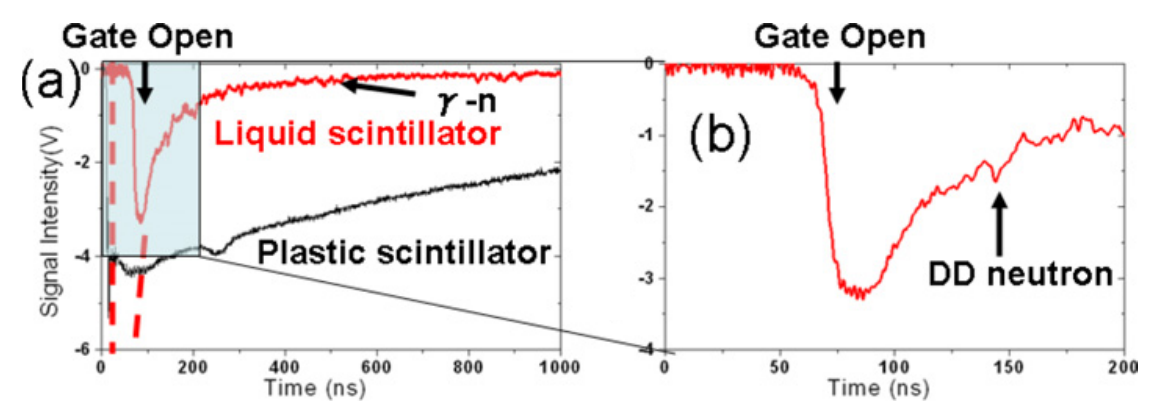

Figure 6. (a) Signals detected by conventional neutron detector and the liquid scintillator detector for the same fast ignition shot. (b) DD neutron signal detected by the liquid scintillator detector. The LFEX laser had an energy of $\sim 300 \mathrm{~J}$. The neutron yield was confirmed to be $3.5 \times 10^{7}$.

around the fusion neutron pulse, as shown in Fig. 6. Thus, the neutron signal should be analyzed in a limited region rather than integrating the signal from the rising edge to the end of the scintillation curve. The absolute sensitivity of the detector was calibrated for exploding pusher target shots. The deuterium (DD) fusion proton generated simultaneously was detected using a CR-39 detector. Only two shots were available, and thus, the error was relatively large in this calibration. The neutron yield detected by the liquid scintillator was also cross-checked via comparison with other calibrated neutron diagnostics.

Figure 6 shows the signal detected for the same fast ignition shot (a) by a conventional plastic scintillator neutron detector and by the newly developed detector and (b) a DD neutron signal detected by the newly developed detector. The LFEX laser had an energy of $\sim 300 \mathrm{~J}$. The signal in the conventional detector was saturated by the strong $\gamma$-rays. On the other hand, the gating in the liquid scintillator successfully blocked the $\gamma$-rays, and the background level was suppressed to the confirmed pulse linearity region. However, a large number of $\gamma$-n signals were included under the blip of the DD neutron. These background signals can be shielded by using a neutron collimator. Details about the analysis of background signals and the estimation of neutron yield have been published in a different paper [10]. The ion temperature from neutron Doppler broadening was not measured using this liquid scintillator because the system response is insufficient for this purpose; the scintillator should be thinner for this to be achieved. In the future, we will install a neutron collimator in the fast ignition system, and we will measure the ion temperature using an improved detector having a thinner liquid scintillator.

\section{SUMMARY}

A fast-decay and low-afterglow liquid scintillator was developed for fast ignition experiments at the GEKKO XII and LFEX lasers at the ILE. The liquid scintillator was coupled to a gated PMT, and the gating performance under high-intensity $\gamma$-rays was experimentally checked. In 2010 , a detector with a high detection efficiency of $10^{-4}$ was developed and installed in the fast ignition experiment. The absolute sensitivity was experimentally calibrated. The neutron yield in the fast heating experiment was successfully detected using this detector.

\section{References}

[1] R. Lauck et al., IEEE Trans. Nucl. Sci. 56, 989 (2009)

[2] C. Stoeckl et al., Rev. Sci. Instrum. 81, 10D302 (2010)

[3] Hamamatsu Photonics K.K.: Photomultiplier Tube, p. 111 [http://jp.hamamatsu.com/resources/ products/etd/pdf/PMT_handbook_v3aJ.pdf] [in Japanese] 


\section{IFSA 2011}

[4] Hamamatsu Photonics K. K. homepage [http://www.hamamatsu.com/]

[5] N. Miyanaga et al., J. Phys. IV France 133, 81 (2006)

[6] Hamamatsu Photonics K.K.: Photomultiplier Tube, p. 151

[7] T. Nagai et al., Jpn. J. Appl. Phys. 50, 080208 (2011)

[8] R. A. Lerche, IEEE. 1, 167 (1991)

[9] L. J. Cline Love et al., Anal. Chem., 48 (4), 364A (1976)

[10] Y. Arikawa et al., Rev. Sci. Instrum. 83, 10D909 (2012) 